Ann. Biol. anim. Bioch. Biophys., I975, 15 (4), 725-738.

\title{
IMPORTANCE \\ DES DIFFÉRENTES PÉRIODES OVOGÉNÉTIQUES DANS LA GONADE FEMELLE D'EMBRYON DE BREBIS; CONTRÔLE DU CHANGEMENT DE COMPORTEMENT MITOTIQUE EN MÉIOTIQUE
}

\author{
P. MAULEON \\ Station de Physiologie de la Reproduction, \\ Centre de Recherche de Tours, I. N.R. A., \\ Nouzilly, 37380 Monnaie
}

avec la collaboration technique de Irrançoise Berthelot et Jacqueline Bezard

\section{RÉSUMÉ}

Les cellules germinales de la gonade d'embryon de brebis qui commencent leur méiose au début, au milieu, ou à la fin de la période ovogénétique $\left(52-82^{\mathrm{e}}\right.$ jours $)$ peuvent participer à la constitution de la réserve ovocytaire trouvée dans l'ovaire de roo jours : injectée à chacun des jours de cette période, la thymidine tritiée s'incorporant pendant la synthèse préméiotique marque fortement des ovocytes qui débutaient alors leur méiose. La proportion variable d'ovocytes provenant de chacun de ces jours est maxima pour les périodes $54-56^{\mathrm{e}}$ jours et $62-64^{\mathrm{e}}$ jours ; cette proportion dépend :

I) des nombres de cellules qui vont se trouver en synthèse préméïotique chaque jour, c'est-àdire du schéma de multiplication des ovogonies. La forme de la courbe du pourcentage d'ovocytes marqués et les variations de fréquence des ovocytes très marqués (index de marquage $>4$ ) en fonction des jours d'injection s'expliquent par un renouvellement des générations de multiplications ovogoniales;

2) de l'intensité des phénomènes de dégénérescence qui détruisent la majeure partie des ovocytes provenant des générations de multiplications débutant après le $66^{\mathrm{e}}$ jour.

Au cours de la période de préméiose, il existe une phase critique (47-50 jours chez 1'embryon de brebis) après laquelle le déroulement de la méiose in vitro se fait normalement mais avant laquelle les modifications créées par la mise en culture suppriment l'apparition des figures méiotiques. Les cellules germinales qui séjournent in vitro gardent la potentialité de reprendre l'enchaînement des processus qui induisent la méïose (greffes d'ovaires cultivés ro jours chez des embryons hôtes). Ces résultats et ceux des travaux récents sur ce sujet sont discutés en face des hypothèses d'environnements local ou général favorables à l'induction de la méïose et d'échanges entre cellules germinales et cellules somatiques. 
Chez les femelles de Mammifères, au cours de la vie prépubère puis adulte, une réserve limitée de gamètes est régulièrement gaspillée. Le nombre d'ovocytes qui constituent cette réserve est très variable. Leur formation dépend des facteurs qui contrôlent le début de la méïose et son arrêt au stade diplotène. Ia période de production de ces ovocytes, ou ovogenèse, a une durée variable suivant les espèces ; elle se termine chez l'embryon de brebis au IOo jour de la gestation (MaUléon, I96I $a$, I967 $a$ I967 b). Le changement de comportement mitotique en comportement méiotique est observable chez des ovogonies entre le $52^{\mathrm{e}}$ et le Ioo $^{\mathrm{e}}$ jour. Toutefois, beaucoup de ces cellules germinales débutant cette différenciation sont détruites lorsque s'opère le blocage de la méiose au stade diplotène. Existe-t-i1 une participation égale de chaque période où débute la méiose à la constitution de la réserve d'ovocytes au stade diplotène? L'entrée en méiose des cellules germinales de la gonade femelle embryonnaire ou post-natale est-elle un phénomène " décidé " parce que transcrit dans la cellule elle-même; cette décision est-elle modifiable ou annulable?

Ce sont ces deux aspects qui ont fait l'objet de ce travail utilisant la technique de marquage cellulaire et celle de culture organotypique.

\section{I. - PARTICIPATION DE CHAQUE PÉRIODE DE, L'OVOGENÈSE

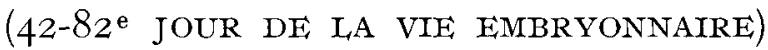

\section{A IA CONSTITUTION DE LA RÉSERVE OVOCYTAIRE DE IA BREBIS}

Les ovocytes formés chez l'embryon sont les mêmes que ceux trouvés chez l'animal prépubère ou adulte (souris : RUDKIN et GRIECH, I96I et I962). Le marquage du noyau des cellules germinales avec la thymidine tritiée pendant les synthèses mitotique et préméiotique d'ADN a permis de le montrer.

\section{Matériel et méthodes}

En faisant varier le jour d'injection de la thymidine tritiée, pendant la période ovogénétique, une image "instantanée " de la population des cellules germinales se multipliant ou débutant leur méiose est fournie par l'étude du marquage autoradiographique des cellules un temps court ( 2 heures) après l'injection. Les variations du pourcentage d'ovocytes marqués et de l'intensité du marquage de ces cellules à la fin de l'ovogenèse ( roo jours) révèlent la part relative jouée par chaque période de l'ovogenèse dans la constitution de la réserve ovocytaire.

Les conditions d'injection de la thymidine tritiée aux embryons, de leur prélèvement ( 2 heures post injection et à l'âge de Ioo jours), de réalisation technique des autoradiographies et aussi d'analyse quantitative du marquage des cellules germinales ont été précisées dans une publication antérieure (MAULÉON, I974). Un soin particulier a été apporté au choix des coupes ou des portions de coupes d'ovaires analysées. L'échantillonnage " raisonné " utilisé a tenu compte pour le choix des coupes de l'hétérogénéité entre coupes de la structure ovarienne : les comptages n'ont eu lieu que sur des coupes comportant à la fois cortex et médulla. Lorsqu'une partie seulement des cellules germinales a fait l'objet de mesure (intensité de marquage) ou d'identification (gonies, ou stades de la prophase méiotique), pout le choix des portions de coupes analysées on a considéré la localisation particulière de chaque type cellulaire : un découpage de zones radiales a été fait sur deux diamètres perpendiculaires. Les corrections de marquage dues au bruit de fond ont été faites à partir de valeurs individuclles cléterminées au-dessus du cytoplasme proche du noyau où était effectuée la mesure d'intensité. Cette mesure, exprimée en quantité de lumière réfléchie par les grains d'argent de l'autoradiographie MPV Leitz), est rapportée à l'unité de 
surface du noyau et donne l'index de marquage $I_{M}=\frac{I_{M P V}-I_{B F}}{S_{N}}$. Dans la détermination des pourcentages moyens de cellules marquées, la valeur moyenne du bruit de fond a été considérée comme égale à 3 ou 4 grains par noyau et la classification d'un ovocyte en marqué ou non marqué a été faite par observation du nombre de grains au-dessus de son noyau.

\section{Résultats}

L’image " instantanée » de la fréquence des cellules germinales qui vont se diviser ou débuter leur méïose est révélée par le taux d'ovogonies et d'ovocytes au stade préleptotène marqué deux heures après 1'administration de thymidine tritiée. Après cette injection, 30 p. Ioo des ovogonies et 32,6 p. Ioo des stades préleptotène-leptotène sont identifiés " "marqués ". Les variations de ces deux paramètres sont respectivement de 25 à 35,5 p. Ioo et de 26,6 à 34,2 p. Ioo selon l'âge de l'embryon le jour de l'injection. Dans le cas des ovogonies, il y a 34,4 p. Ioo des cellules germinales marquées lorsque l'injection est faite avant le $64^{\mathrm{e}}$ jour de gestation et $26,3 \mathrm{p}$. Ioo si elle est faite après. Cette différence est significative. Il n'y a qu'une très faible variation selon ces deux périodes de l'ovogenèse dans le taux de préleptotènes marqués (moins de 4 p. Ioo).

Lorsque le prélèvement est effectué à la fin de l'ovogenèse (roo jours), le taux d'ovocytes marqués est mesuré un temps variable après l'incorporation de thymidine tritiée, mais ce temps est toujours supérieur à I 2 jours, c'est-à-dire à la durée de la méïose du stade préleptotène au stade diplotène. Dans ces conditions, par suite de la dilution du marquage au cours des divisions, seuls seront " identifiés marqués " les ovocytes qui ont incorporé la thymidine tritiée dans leur ADN au cours de la synthèse préméïotique ou au cours d'une synthèse mitotique qui n'a pas été suivie de plus de deux (ou trois) divisions.

La courbe de fréquence du pourcentage d'ovocytes marqués $\left(\mathrm{P}_{100}\right)$ à un âge donné est donc l'image transposée dans le temps de la fréquence des cellules germinales passant d'un comportement mitotique à un comportement méiotique ou étant sur le point de le faire, ce que nous conviendrons d'appeler avec CHal,oner (I975) en préméiose. Or, nous avons vu qu'en première approximation, environ le $I / 3$ de toutes les cellules se trouvant dans cette situation incorpore le radio-élément quel que soit le jour de l'injection. S'il existe de fortes variations de $\mathrm{P}_{100}$ en fonction de ce jour, elles seront donc le reflet des variations numériques des cellules germinales en préméiose. Cette image transposée est toutefois modifiée par les phénomènes de dégénérescence au moment de l'arrêt au stade diplotène. Ces phénomènes sont intenses à partir du $65^{\mathrm{e}}$ jour d'âge. De nombreux ovocytes marqués et non marqués disparaissent alors selon une règle que nous ne connaissons pas.

\section{Phases de la période ovogénétique participant à la constitution de la réserve ovocytaire.}

En dépit de la complexité du critère $P_{100}$ qui est la résultante de plusieurs phénomènes (rythmes des divisions ovogoniales, asynchronisme des évolutions, renouvellement des générations de multiplication, disparition des cellules), la forme de la courbe de fréquence de ce pourcentage d'ovocytes $\left(\mathrm{P}_{100}\right)$ permet de répondre à cette question.

Une forte proportion de la réserve ovocytaire provient de la période ovogéné- 
tique située entre le $5^{2}$ et le $64^{\mathrm{e}}$ jour, puisque, si l'injection est faite un de ces jourslà, de 45 à 70 p. Ioo des ovocytes sont identifiés marqués dans l'ovaire de Ioo jours.

Les ovocytes qui sont les premiers à entrer en méïose à 52-54 jours se retrouvent comme éléments constituant le stock de gamètes aussi bien que ceux qui débutent leur méiose le $72^{\mathrm{c}}$ jour; mais ils seront peu nombreux.

\section{TABLEAU I}

Fréquence (en $\mathrm{p}$. Ioo) par classes d'index de marquage des noyaux des ovocytes
selon l'áge de l'embryon de brebis le jour d'injection de la thymidine tritiée

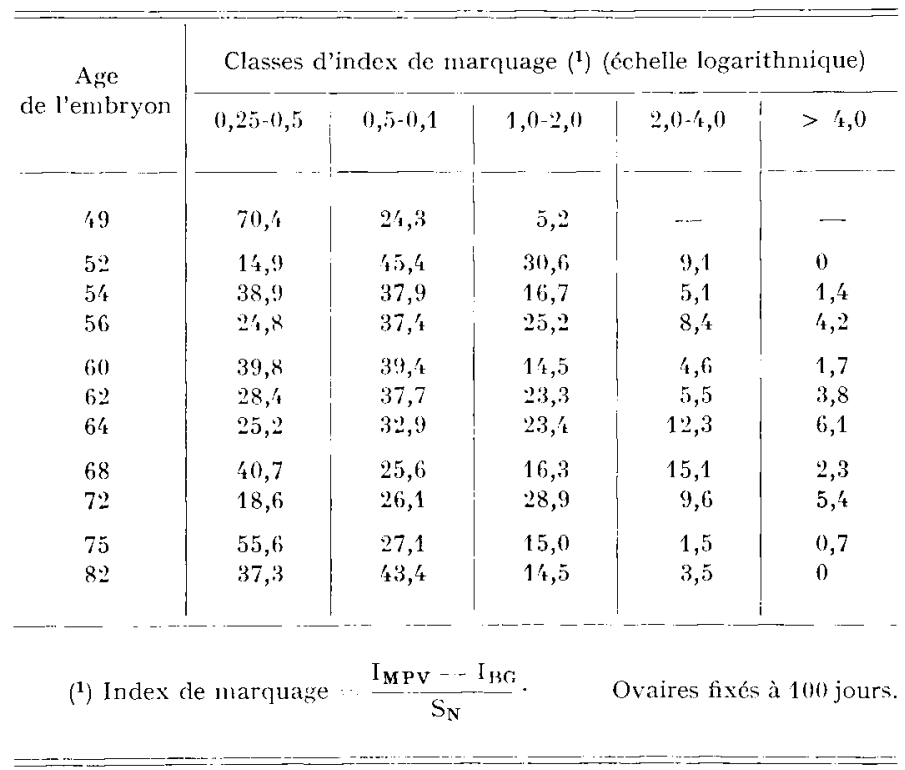

Si l'on considère les ovocytes fortement marqués (index de marquage supérieur à 4 , tabl. I), une conclusion identique peut être tirée : du début à la fin de la période ovogénétique les ovocytes néoformés participent à la constitution de la réserve ovocytaire. Ces ovocytes fortement marqués ( $\mathrm{I}_{M}>4$ ), c'est-à-dire ceux dont l'injection de thymidine tritiée a révélé la synthèse préméïotique, sont localisés près de la médulla dans l'ovaire de roo jours lorsque l'injection est faite le 52-56e jour, au milieu du cortex pour le $64^{\circ}$ et très superficiellement après administration le $72^{\mathrm{e}}$ jour. Dans la couronne corticale ovarienne qu'ils peuplent, les ovocytes à cet âge peuvent être datés par la localisation topographique. Ce fait est clair chez la brebis car les séquences de leur formation s'étalent sur un temps assez long (P1. I).

Il existe bien une période ovogénétique qui contribue plus que les autres à l'établissement de la réserve, comme le suggéraient BLAck et ErRICKson (Ig68) chez le porc. 'Toutefois, elle reste large et l'avantage d'avoir étudié chaque jour de cette période et non pas quelques points espacés (BIACK et ErICKson, I968) est de faire apparaître que chez la brebis l'ovogenèse est un processus qui doit être entretenu pour être capable de durer un temps aussi long. 
2. Existence d'au moins deux générations de multiplications ovogoniales chez l'embryon de brebis.

La courbe de fréquence du pourcentage d'ovocytes marqués $\left(\mathrm{P}_{100}\right)$ ne présente pas un plateau entre les jours d'injection 52 et 64 , mais une chute marquée vers le $60^{e}$ jour. La valeur $P_{100}$ pour l'injection à 60 jours $(46,8$ p. Ioo $\pm 3,6)$ est significativement différente des deux maxima, 64,8 et 68,2 , qui l'encadrent pour des injections à $J_{54-56}$ ou $J_{62-64}$. Un plus faible pourcentage d'ovocytes très marqués $\left(I_{M}>4\right.$ ou $\left.I_{M}>2\right)$ est trouvé dans l'ovaire de Ioo jours si l'injection est faite à $J_{60}$ au lieu de $\mathrm{J}_{56}$ et $\mathrm{J}_{64}$ (respectivement $\mathrm{I}, 7 \mathrm{p}$. Ioo et $6,5 \mathrm{p}$. Ioo vs; 4,2 p. Ioo et I2,6 p. IOo; 6 ,I p. Ioo et I 8,4 p. IOO).

Cette période centrée à $\mathrm{J}_{60}$ est donc encadrée, d'après ces résultats, par deux moments où de plus grands nombres de cellules germinales sont en " préméïose ". C'est ce que nous avons confirmé expérimentalement, en déterminant dans les ovaires de ces différents âges $\left(\mathrm{J}_{60}, \mathrm{~J}_{56}, \mathrm{~J}_{64}\right)$ deux heures après l'injection la fréquence des stades préleptotènes rapportées au nombre total de cellules germinales (I4 p. Ioo $v s, 23$ et 24 p. IOO).

Il est donc logique de penser que les deux paramètres $P_{100}$ et $I_{M}>4$ sont plus faibles lorsqu'une injection a lieu à $J_{60}$ parce que l'on trouve pour une forte proportion de cellules germinales une dilution du marquage identique à celle observée avant le $5^{2}$ jour.

L'hypothèse qu'une nouvelle génération de multiplications ovogoniales à partir de gonies souches a commencé vers $1 \mathrm{e} 60^{\mathrm{e}}$ jour peut-être émise. La plus grande fréquence des ovogonies dans l'ovaire de 62 à 64 jours $(79,4$ et 62,3 p. Ioo) succédant à une diminution régulière de ce pourcentage $d u 5^{2-56^{e}}$ au $60^{\mathrm{e}}$ jour $(98 \mathrm{p}$. I00-76,5 p. IOO : 54,5 p. IOO) appuie cette hypothèse.

Les ovogonies débutant ou approchant du début de la méïose dans l'ovaire de 72 jours se retrouvent peu nombreuses dans l'ovaire de Ioo jours. Pourtant, les ovogonies presqu'absentes à $66-68$ jours $(23,5$ p. Ioo et 26,4 p. Ioo des cellules germinales) redeviennent proportionnellement plus nombreuses que les autres types cellulaires, certes frappés par les phénomènes de dégénérescence à $J_{72}$ et $J_{75}(68,4$ et $55,9 \mathrm{p}$. IOO). Le pourcentage des ovocytes fortement marqués $\left(I_{M}>4\right)$ et $\left(I_{M}>2\right)$ après injection de thymidine à $J_{72}$ est aussi plus élevé qu'après des injections à $J_{68}$ et $\mathrm{J}_{75}$ (tabl. I). Une nouvelle génération de cellules germinales a débuté une série de multiplications vers 66 jours mais l'intensité de la dégénérescence l'a rendue inefficace pour la constitution du stock d'ovocytes.

\section{Discussion}

La comparaison de la courbe de fréquence des pourcentages d'ovocytes marqués (fig. I) avec celle obtenue chez la ratte en suivant une méthodologie d'étude identique (MaulÉon, I967a et b) illustre l'hypothèse qu'il existe chez l'embryon de brebis au moins deux générations de multiplications ovogoniales identiques à celle mise en évidence chez la ratte. Ces générations se succèdent mais s'enchevêtrent par suite de l'asynchronisme d'évolution des cellules germinales appartenant à une même génération. 
De même, 1'hétérogénéité de générations auxquelles appartiennent les cellules germinales qui incorporent la thymidine tritiée au moment de l'injection tessort également de la comparaison des distributions des fréquences d'intensité de marquage à un âge donné (comparaisons faites après transformation de LAZAR et GERARDMarchand, I 962). Lorsqu'il y a seulement asynchronisme d'évolutions entre cellules, la courbe des fréquences cumulées (échelle probit-log.) est une droite (cas de la ratte) (MAULÉon, I967 $a$ et $b$ ). S'il existe plusieurs générations cellulaires décalées dans leur évolution, cette courbe est une ligne brisée (MaULÉon, 1974).

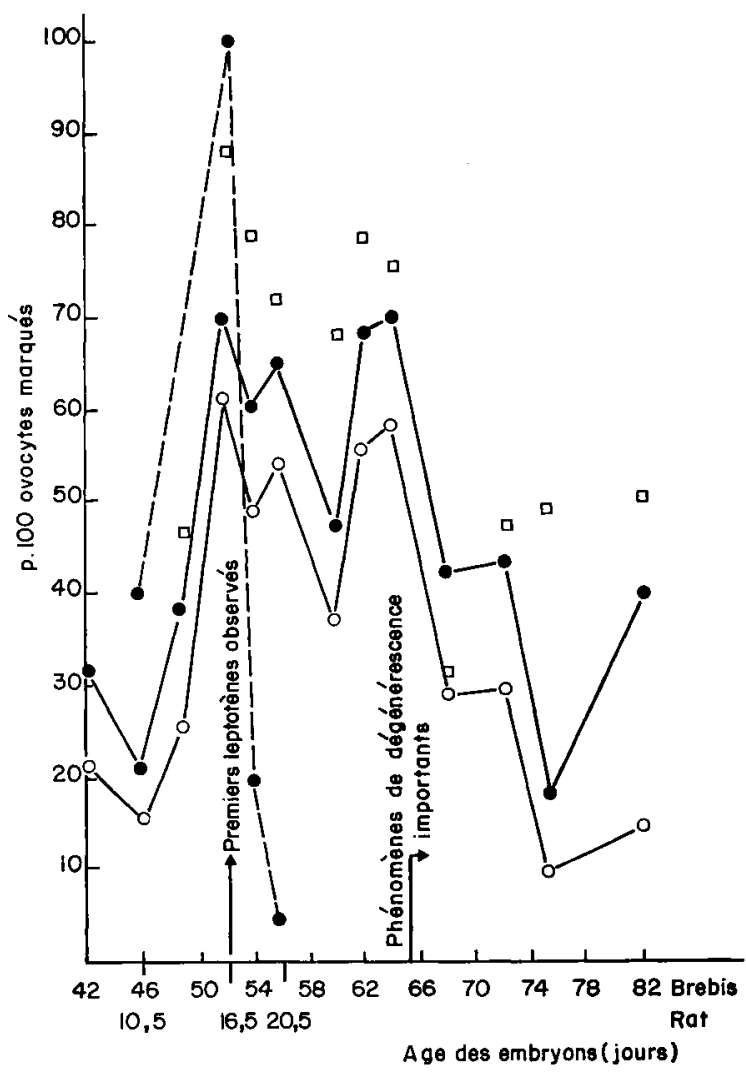

FIG. I. - Variations des pourcentages d'ovocytes marqués

- dans l'ovaire d'cmbryon de brebis de Ioo jours, $\square$ index de inarquage $<0,25$ au MPV $\bullet-\bullet$ si plus de 4 grains

dans l'ovaire de rat de 20 jours après la naissance,

- - $\ldots$ si plus de ro grains

en fonction du jour d'injection du narqueur radioactif (thymidine tritiée).

La forme de la courbe de fréquence des mitoses goniales bloquées pendant 3 heures par la colchicine ne pouvait aussi s'expliquer qu'en faisant la même hypothèse (MAUlÉon, I96I b). 


\section{II. - EXISTENCE D'UN CONTRÔLE DU DÉBUT DE LA MÉÏOSE}

\section{DANS LES OVOGONIES D'OVAIRES D'EMBRYON DE BREBIS}

I,entrée des cellules germinales femelles en méîose est un événement " décidé " par leur constitution génétique et que rien ne vient perturber. Cette opinion souvent exprimée s'appuie sur le fait que " le processus entier de l'ovogenèse peut avoir lieu in vitro dans des conditions de culture appropriées" (MARTINOviTCH, I938). Flle est confirmée par les résultats obtenus avec des chimères expérimentales $\mathrm{XX} / \mathrm{XY}$ de souris (Mystowska et TARkowskr, I970; McLAREN et al., I972) : les cellules germinales qui entrent en méiose dans la gonade avant la naissance sont des cellules $\mathrm{XX}$.

Nous avons essayé de voir si des modifications expérimentales de l'environnement des cellules germinales au cours de leur mise en culture in vitro ne pouvaient pas empêcher le changement de comportement mitotique en méiotique de s'opérer dans les gamètes femelles. Nous ne nous sommes donc intéressés qu'au début de la méiose, c'est-à-dire à sa première phase, les autres phases (formation du complexe synaptinemal, évolution au-delà de ce stade, arrêt du mécanisme de la prophase méiotique) pouvant dépendre de contrôles différents.

\section{Matériel et méthodes}

Les techniques de culture organotypique sont celles précisées précédemment (MAULÉon, I973). Dans les cultures immergées, les gonades reposent sur un fond gélosé (I p. Ioo dans solution de Earle), le milieu de culture étant de même composition que celui utilisé pour les cultures sur grille. La technique de greffe des ovaires cultivés in vitro chez les embryons hôtes n'a reçu que des modifications mineures du système de fixation du greffon par rapport à celle de MAULÉon (I973). L'analyse cytologique des stades la prophase méiotique a été faite par la méthode de LucIANi et al. (1974).

\section{Résultats}

\section{Infuence de l'âge de l'embryon au moment du prélèvement de l'ovaire pour sa mise} en culture sur le déroulement de la méiose.

Le changement de comportement mitotique en comportement méiotique ne se produit pas en culture organotypique lorsque la gonade est prélevée avant le $50^{\mathrm{e}}$ jour de la vie embryonnaire. Les premières figures de début de la méïose apparaissent normalement au $52^{\circ}$ jour in vivo (sur moins de 2 p. Ioo des cellules germinales).

L'évolution de la prophase méïotique du stade leptotène au stade diplotène se poursuit dans les cultures organotypiques réalisées dans les mêmes conditions techniques lorsque l'ovaire d'embryon de brebis est prélevé après le $5^{\text {e }}$ jour de la vie. Toutefois, l'étude quantitative des proportions de stades de début de méiose (leptotène + zygotène) par rapport aux stades de fin de méîose (pachytène + diplotène) montre qu'un faible nombre d'ovogonies entre en méiose après la mise en culture. 
Comme nous l'avons déjà discuté précédemment (MaUlÉoN, I973), ces résultats n'étaient pas en contradiction avec la plupart des observations des auteurs ayant publié auparavant puisque presque tous avaient mis en culture des ovaires présentant déjà les premières figures de méïose (tabl. 2).

\section{TABLEAU 2}

Age des embryons au moment de l'explantation de la gonade pour sa mise en culture.

Classement des prélèvements selon les résultats du déroulement de la méïose in vitro

\begin{tabular}{|c|c|c|c|c|}
\hline \multicolumn{2}{|c|}{ Espèce } & \multirow{2}{*}{$\begin{array}{c}\text { Age de l'embryon } \\
\text { au moment de la mise } \\
\text { en culture (jours) } \\
\\
12-13 \\
16 \\
13-18\end{array}$} & \multirow{2}{*}{$\begin{array}{l}\text { Déroulement } \\
\text { de la méiose } \\
\text { in vitro }\end{array}$} & \multirow{2}{*}{$\begin{array}{l}\text { Auteurs } \\
\text { WOLFF (1952) } \\
\text { BLANDAU et al. (1965) } \\
\text { ODOR et BLANDAU (1971) } \\
\text { BORGHESE et VENINI (1956) }\end{array}$} \\
\hline Souris & $(13 j)$ & & & \\
\hline$R a t$ & $(16,5-17 j)$ & $\begin{array}{l}15-16 \\
2 \mathrm{j} \text { p.n. }\end{array}$ & Normal & $\begin{array}{l}\text { Martinovitch (1936) } \\
\text { Bousguet }(1967)\end{array}$ \\
\hline $\begin{array}{l}\text { Hamster } \\
\text { Brebis } \\
\text { Homme }\end{array}$ & $\begin{array}{l}(1 \mathrm{j} \mathrm{p} . n .) \\
(52 \mathrm{j}) \\
(8 \mathrm{sem} .)\end{array}$ & $\begin{array}{c}15 \\
55 \\
9-28 \text { sem. } \\
22 \mathrm{~mm}-171 \mathrm{~mm}\end{array}$ & & $\begin{array}{l}\text { Challoner (1975) } \\
\text { MaulÉon (1973) } \\
\text { Baker et Neal, (1974) } \\
\text { Blandau }(1969)\end{array}$ \\
\hline Ponlet & $(16 \mathrm{j})$ & $8-10-12$ & jusqu'à lepto-zygo & ERICKSON (1974) \\
\hline $\begin{array}{l}\text { Poulet } \\
\text { Brebis } \\
\text { Hamster } \\
\text { Souris }\end{array}$ & $\begin{array}{l}(16 \mathrm{j}) \\
(5 \div \mathrm{j}) \\
(1 \mathrm{j} \text { p.I..) } \\
(13 \mathrm{j})\end{array}$ & $\begin{array}{c}4-6 \\
47-50 \\
13-1 / t \\
11\end{array}$ & Absence & $\begin{array}{l}\text { İRICKSON }(1974) \\
\text { MAUĹ́on }(1973) \\
\text { CHALLONER }(1975) \\
\text { WOLFF }(1952)\end{array}$ \\
\hline
\end{tabular}

( ) Age de l'embryon au moment de l'apparition des premières figures de prophase méiotique pour chaque espèce.

Les observations de SalzGeber (I962) et d'OGawa (I968) à la suite de mise en culture de très jeunes ovaires de souris pourraient être une confirmation des résultats que nous obtenons. Les mauvaises conditions de prélèvement des gonades indifférenciées et de petite taille peuvent être jugées responsables de l'absence de figure de méiose dans les gonades en culture ; cette hypothèse a d'ailleurs été formulée par ERICKsON (I974) observant ces mêmes résultats après prélèvement de gonades d'embryon de poulet avant le $8 \mathrm{e}$ jour.

Deux précisions doivent être apportées aux conclusions telles que nous avons pu les résumer ci-dessus :

a) Le pourcentage de cellules en début de méïose (formes anormales citées par MAULF́ON, I973, ou leptotène-zygotène d'ERICKSON, I974) augmente lorsque l'âge de prélèvement s'approche de celui auquel apparaissent normalement les premières figures de méíose. 
Le pourcentage de cellules en début de prophase méiotique par rapport aux cellules germinales présentes dans l'ovaire double lorsque la gonade analysée à un même âge de 58-60 jours est prélevée à 50-52 jours au lieu de $47-49$ jouts (MaULÉon, I973). De même, dans les gonades d'embryon de poulet mises en culture à 8 jours, il y a de 2 à 60 p. Ioo de cellules en début de méiose à $I_{7}$ jours, mais de 87 à 96 p. roo si ces gonades le sont à I2 jours (ERICKSON, I974).

b) Dans les figures de début de méiose présentées dans les publications, lorsque 1'âge de prélèvement est proche bien qu'antérieur à celui de 1'âge normal d'apparition des premiers leptotènes, l'appariement des filaments chromosomiques n'a jamais été noté. L'examen histologique de ce point de vue est nettement insuffisant et seule une étude cytologique permet de 1'affirmer (Pl. II et III).

Nous avons parlé (MAUl,́́on, I973) ainsi que Chal,Loner (I975), de formes anormales. Ces formes (leptotènes "en boule ") sont égalemant rencontrées à la fin de la période ovogénétique normale ( 80 jours), période pendant laquelle beaucoup des cellules en début de méiose ne vont pas évoluer. Il nous a somblé aussi, comme cet auteur, observer histologiquement des stades zygotène que nous n'avons pas retrouvés au cours des études cytologiques. Nous ne pensons pas également que les figures cytologiques interprétées comme zygotène dans le travail d'ERICKSON (I974) montrent d'appariements chromosomiques.

\section{L'état des cellules germinales après 10 jours de culture est tel qu'il est possible de faire réapparaître la méiose dans ces collules.}

Les conditions de survie in vitro sont souvent considérées comme défavorables. Pourtant, le déroulement de la méïose in vitro, lorsqu'elle a débuté, se fait de façon identique à celui réalisé $i n$ vivo. Dans l'ovaire humain, la durée des stades de la méiose est la même : leptotène, 2 jours ; zygotène, Io jours ; pachytène, 20 jours (BAKER et NEAL, I974).

De plus, les résultats que nous avons obtenus ne dépendent pas des méthodes de culture et sont identiques après culture sur grille ou culture immergée selon une technique voisine de celle d'ERICKSON (I974). Chez le hamster, si les ovaires sont explantés avant le $I 5^{\mathrm{C}}$ jour p. c., la prophase méiotique ne commence pas après une culture de la gonade par une méthode identique à celle de BAKER et NEaL (rg69), donc très différente de la nôtre par son milieu, les proportions de $\mathrm{CO}_{2}$ et la pression du gaz environnant (CHALLONER, I975).

Les conditions de culture in vitro ne sont sans doute pas trop déprimantes pour les cellules germinales puisqu'une reprise de la méiose a été observée dans les ovaires d'embryons de brebis mis en culture depuis ro jours après greffes chez des embryons hôtes (MAULÉoN, I973).

Deux remarques doivent être faites sur ces résultats de greffe. Elles orientent les hypothèses que l'on peut faire sur l'absence d'apparition de la méïose in vitro dans les ovaires explantés à un âge "critique " :

a) La reprise de la méiose après greffe n'a été observé que dans un nombre limité de cas très précis dépendant de l'âge de l'embryon hôte (tabl. 3) ; la survie même des cellules germinales indépendamment de la reprise de la méiose est toujours meilleure chez des embryons jeunes. Les greffons ont été placés après castration de l'embryon dans cette même localisation gonadique. Chaque fois qu'un fragment 
d'ovaire de l'embryon hôte a été oublié ou laissé volontairement son identification a toujours été facile et aucune confusion n'est possible entre un tel fragment et le greffon de l'ovaire provenant de la culture in vitro.

TABIEAU 3

Résultats des greffes d'ovaives d'embryons de brebis cultivés in vitro pendant 9-10 jours chez des embryons hôtes de la même espèce

\begin{tabular}{|c|c|c|c|c|c|c|c|c|c|c|c|}
\hline & \multirow{3}{*}{ Age } & \multirow{7}{*}{$\begin{array}{c}\text { Nbre } \\
\text { ovaires }\end{array}$} & \multicolumn{9}{|c|}{ Résultats } \\
\hline & & & \multicolumn{9}{|c|}{ Embryons vivants } \\
\hline & & & \multirow{5}{*}{$\begin{array}{c}\text { Embryons } \\
\text { morts }\end{array}$} & \multirow{5}{*}{$\begin{array}{l}\text { Greffons } \\
\text { non } \\
\text { retrouvés }\end{array}$} & \multicolumn{7}{|c|}{ Greffons retrouvés } \\
\hline \multirow{4}{*}{$\begin{array}{l}\text { Ovaire } \\
\text { mis en } \\
\text { culture }\end{array}$} & \multirow{4}{*}{ Embryon hôte } & & & & \multicolumn{7}{|c|}{ Présence de cellules germinales } \\
\hline & & & & & \multirow{3}{*}{$\begin{array}{c}\text { Pas de } \\
\text { cellules } \\
\text { germinales }\end{array}$} & \multicolumn{3}{|c|}{$\begin{array}{l}\text { Absence } \\
\text { de méiose }\end{array}$} & \multicolumn{3}{|c|}{$\begin{array}{l}\text { Présence } \\
\text { de méiose }\end{array}$} \\
\hline & & & & & & \multicolumn{6}{|c|}{ Durée de séjour chez l'hôte } \\
\hline & & & & & & & 10 & & 12 & 14 & \\
\hline $37 \mathrm{j}$ & $56-58 j$ & 3 & $0 / 3$ & $0 / 3$ & $0 / 3$ & & & 3 & & & \\
\hline $47-49 \mathrm{j}$ & $\int 56-58 j$ & 19 & $2 / 19$ & $3 / 17$ & $0 / 1^{\prime}$ & 4 & 5 & 1. & 1 & $\mathbf{2}$ & 1 \\
\hline $47-43 \mathrm{~J}$ & $120 j$ & 10 & $2 / 10$ & $2 / 8$ & $5 / 6$ & 1 & & & & & \\
\hline
\end{tabular}

Une première hypothèse est que cette reprise résulte d'un environnement hormonal favorable lié à l'âge de l'embryon hôte. Elle est en contradiction avec les résultats négatifs obtenus par addition d'hormones sous diverses formes au milieu de culture (Mauléon, I973; Chaldoner, I975). Toutefois, toutes les hormones n'ont pas été essayées et la réalisation d'un équilibre hormonal optimum est toujours aléatoire in vitro.

Une deuxième hypothèse, celle de l'existence d'une substance diffusible émise localement par la gonade (ou un autre organe) de l'embryon hôte pourrait être faite après l'observation que les résultats positifs sont obtenus dans des gonades greffées près du mesonephros. Cette hypothèse a d'ailleurs été faite par ERICKSON (I974) cultivant des cellules isolées avec des fragments de cortex. Toutefois, les cultures associées d'ovaires d'embryons de brebis prélevés avant et après le $50^{\circ}$ jour montrent une évolution indépendante et caractéristique des gonades explantées à deux âges différents (P1. IV).

b) Un temps de séjour de I2 jours chez 1'hôte est nécessaire pour que la méïose soit observée dans le greffon; il faut admettre qu'il existe dans 1'ovaire "cultivé " des ovogonies capables d'entrer en méïose et que la greffe permet non pas une simple levée d'inhibition d'un phénomène, mais la mise en place d'un enchaînement de processus.

\section{Discussion}

Des résultats précédents, il apparaît :

I. Un moment critique avant lequel les ovogonies sont incapables d'entrer en méiose in vitro. 
Entre 47-49 jours chez l'embryon de brebis (MAulÉon, I973), I4-I5 jours chez le Hamster (Challoner, I975), II-I2 jours chez la Souris (d'après les remarques de WolfF, I952, et SAlzGEBER, I962) et sans doute I3-I4 jours chez le Rat.

Cet état mal défini actuellement ne se situe pas immédiatement avant la dernière synthèse préméïotique d'ADN mais plutôt pendant une "préméiose " où les cellules ont encore 111 comportement mitotique.

2. Un environnement local favorable doit être nécessaire à un apport (actuellement hypothétique) de substances " trophiques " nécessaire à l'induction de la méiose.

Récemment, Byskov (r974) a montré le rôle possible des cellules somatiques du rete ovarii dans une telle induction en supprimant leur présence avant la greffe d'ovaires chez des souris hôtes immunologiquement non réactives. Beaucoup d'imprécisions existent dans les publications sur les conditions de séparation des ovaires et des tubules du rete ovarii avant la mise en culture des gonades. Dans nos expériences, la taille de l'ovaire d'embryon de brebis avant le $5^{\circ}$ jour et son mode de rattachement au mesonephros permettent de le disséquer parfaitement. Une telle dissection est plus difficile à réaliser sans précautions particulières avec un ovaire très jeune đ'embryon de rat ou de souris. Dans le cas de l'ovaire de brebis, les premiers tubules du rete ovarii sont cependant observables à la liaison gonade-mésonéphros dès le 28 jour (MAUlÉon, I975). Le type de dissection que nous avons opéré ne ressemble pas à 1'élimination totale permise par une localisation extra-ovarienne des tubules du rete ovarii d'embryon de souris de I2 jours telle que l'a pratiquée Byskov (I974). Nous ne pouvons cependant pas nier l'importance qu'une suppression involontaire, même partielle de la colonisation de la gonade par les cellules du rete ovarii a pu avoir dans les résultats que nous avons observés. A 47 jours, ces cellules peuvent n'avoir atteint que les gonies les plus internes et ce sont celles qui survivent le moins bien aux cultures organotypiques.

L'existence des relations cellules somatiques-cellules germinales expliquerait que, dans le cas des chimères expérimentales, peu de cellules germinales XX entrent en méiose : elles ne pourraient le faire que sous l'influence d'un tissu somatique XX proche. Toutefois, actuellement, on ne peut pas dire si de tels effets des cellules .somatiques sur les cellules germinales dépendent du contact cellulaire direct ou d'un composé sécrété par les cellules somatiques.

3. Un environnement hormonal général favorable n'est pas une hypothèse à éliminer et une telle action peut être indirecte : les échanges des cellules somatiques du rete ovarii sont peut-être modifiables par un tel environnement aussi bien que le taux de multiplication de ces cellules, c'est-à-dire leur nombre.

Enfin, les autres phases de la méïose, comme la formation, et une évolution normale du complexe synaptinemal peuvent être plus directement dépendantes de ces facteurs hormonaux. Les expériences entreprises jusqu'ici à ce sujet restent confuses et critiquables chez la femelle. 


\title{
REMERCIEMENTS
}

Nous remercions M. Michel Terriot pour les microphotographies jointes à ce texte. Ce travail a bénéficié de l'aide de la D. G. R. S. T., contrat no 5221 .

\section{SUMMARY}

\author{
IMPORTANCE OF VARIOUS OOGENE'TIC PERIODS IN THE \\ EMBRYONIC SHEEP GONAD :
} CONTROL, OF CHANGING MITOSIS IN MEIOSIS

The germ cells of the embryonic sheep gonad which inter into mejosis at the beginning, in the middle or at the end of the oogenetic period (52-82nd day) may participate in the constitution of the oocyte reserve : when injected at each day during this period, tritiated thymidine incorporated during premeiotic synthesis, strongly labels the oocytes of the roo th day ovary then in a such state. The variable proportion of oocytes originating from each of these days is maximal for the period 54-56 days and 6z-64 days; this proportion depends :

1) on the number of cells in premeiotic synthesis on each day, that is, on the scheme of oogonial mitosis. The shape of the curve of percentage of labelled oocytes and the variations in the frequency of oocytes with a labelling index $>4$, as a function of the day of injection is explained by a renewal of the generations of oogonial multiplication;

2) on the intensity of the degenerativa phenomena which destroy the greater part of oocytes originating from generations of multip'ications begun after the 66th days.

During the period of premeiosis, there is a critical phase $\left(47^{-5}\right.$ o days for the sheep embryo) after which meiosis in vitro occurs normally, but before which, modifications due to culture conditions suppress the appearance of meiotic figures. The germ calls during their in vitro period keep the potential to carry out the chain of reactions which induce meiosis (ovaries cultivated in vitro Io days and grafted in host embryos). These results and those of recent works on this subject are discussed with the hypotheses of local or general environments favourable to the induction of meiosis and exchanges between germ cells and somatic cells.

\section{RÉFÉRENCES BIBLIOGRAPHIQUES}

Baker T. G., NeAL P., I 969 . Effects of $\mathrm{X}$ radiation on mammalian oocytes in organ culture. Biophysik, 6, 39-45.

Baker T. G., NEAL P., 1974. Oogenesis in human fetal ovaries maintained in organ culture. J. Anat., $11 \%, 59 \mathrm{I}-604$.

Black J. L., Erickson B. H., I968. Oogenesis and ovarian development in the prenatal pig. Anat. Rec., 161, 45-56.

Blandau R. J., I969. Observations on living oogonia and oocytes from human embryonic and fetal ovaries. Am. J. Obstet. Gynec., 104, 310-319.

Blandau .R. J., Warrick E., Rumery R. E., I965. In vitro cultivation of fetal ovaries. J. Fertil. Steril., 16, j05 7 I 5 .

Borghese E., VeniNi M. A., 1956. Culture in vitro di gonadi embriovali di mus. musculus. Symposia Genetica, 5, 69-83.

Bousouet J., I967. Culture organotypique de fragments d'ovaires de ratte. Action de facteurs gonadotropes hypophysaires. C. R. Soc. Biol., 161, $778-782$.

Byskov A. G., I974. Does the rete ovarii act as a trigger for the onset of meiosis? Natuve, 252, $396-397$.

Challoner S., 1975. Studies of oogenesis and follicular development in the Golden hamster. II. Initiation and control of meiosis in vitro. J. Anat., 119, I49-I56. 
ERICkson G. F., I974. The control of the differentiation of female embryonic germ cells in the bird. Dev. Biol., 36, II 3 - I 29.

Lazar P., GERaRd-Marchant R., I965. Une technique de représentation graphique des numérations de grains sur auto-historadiographie. Ann. Histochim., 10, 43-52.

Luciani J. M., Devictor-Vuillet M., Cagne R., Stall A., I974. An air-drying method for first meiotic prophase preparations form mammalian ovaries. J. Reprod. Fert., 36, 409-4II

Mclaren A., Chandiey A. C., Kofman-Alfaro S., I972. A study of meiotic germ cells in the gonads of foetal mouse chimaeras. J. Embryol. exp. Morph., 27, 515-524.

Martinovitch P. N., r938. The development in vitro of the mammalian gonad ovary and ovogenesis. Proc. Roy. Soc., Ser. B, 125, 232-249.

MaUleon P., rg6ra. Déroulement de l'ovogenèse comparé chez différents mammifères domestiques. Proc. IVth Cong. intern. Reprod. anim., La Haye, 2, 348-354.

Mauleon P., I96r b. Utilisation de la colchicine dans l'étude des divisions goniales de l'ovaire d'embryon de brebis et analyse de quelques résultats. Ann. Biol. anim. Bioch. Biophys., 1, 7o-73.

Mauleon P., ig67 a. Cinétique de l'ovogenèse chez les mammifères. Archs. Anat. Micr. Morph. exp., 56 (Suppl. 3-4), I25-I56.

Madleon P., I967 b. Oogenesis and folliculogenesis. In Reproduction in Domestic Animals, ColE et Cupps Ed., Acad. Press Publ., 2 e Ed., Chapter 7.

Mauleon P., 1973. Modification expérimentale de l'apparition et de l'évolution de la prophase méiotique dans l'ovaire d'embryon de brebis, Ann. Biol. anim. Bioch. Biophys., 13 (hors série), 89-1o2.

Mauleon P., 1974. The establishment of the primordial follicle reserve in the sheep embryo studied by labelling of oocytes with tritiated thymidine. Europ. J. Obstet. Gynec. Reprod. Biol., 4, Suppl. 4, I33-I39.

Mauleon P., I975. Migratory phase of germ cells and sexual differentiation of the gonad in sheep embryo. Ann. Biol anim. Bioch. Biophys., (A paraître).

Mystowska E. T., TARKowskI A. K., 1970. Behavior of germ cells and sexual differentiation in late embryonic and early postnatal mouse chimeras. J. Embryol. exp. Morph., 23, 295-405.

Odor D. L., Blandau R. J., i971. Organ culture of fotal mouse ovaries. Am. J. Anat., 131, 387-396. OGawa W., Ig68. Studies on the ovogenesis of the mice embryos using tissue culture method. Jap. J. Fert. Steril., 13, 64-78.

Rudkin G. T., Griech H. A., I96I. H33-thymidine labelling of foetal mouse oocyte nuclei. J. Histochem. Cytochem., 9, 62 I (Abstr.).

Rudkin G. T., GRIEch H. A., I962. On the persistance of oocyte nuclei from fetus to maturity in the laboratory mouse. J. Cell. Biol., 12, I69-176.

Salzgeber B., I962. Étude du développement de l'ovaire de souris greffé dans l'embryon de poulet après culture in vitro. Archs Anat. Micr. Morph. exp., 51, I-ro.

Wolf F E., I952. Sur la différentiation sexuelle des gonades de souris explantées in vitro. C. R. Acad. Sc., Paris, Ser. D, 234, I7I2-I7I4. 


\section{PLANCHE I}

Localisations différentes dans le cortex des ovocytes

fortement marqués (ayant incorporé la thymidine tritiée pendant la synthèse d'ADN préméiotique ou proche de ce moment) en fonction du jour d'injection du radioélément

I. Injection le $5^{6 \mathrm{e}}$ jour de la vie embryonnaire. Localisation profonde.

2. Injection le $64^{\mathrm{e}}$ jour de la vie embryonnaire. Localisation moyenne.

3. Injection le $72^{\mathrm{e}}$ jour de la vie embryonnaire. Localisation superficielle.

Ovocytes très marqués localisés par une flèche. Autoradiographie : film Ilford $\mathrm{K}_{5}-\mathrm{Feulgen.}$ 


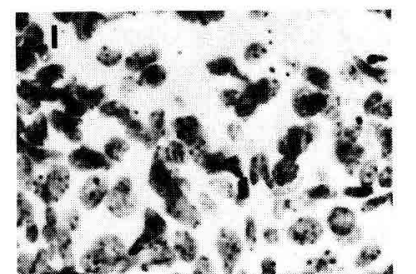

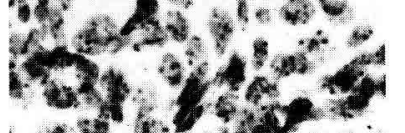
4. $y^{2}+2+3 y^{2}$

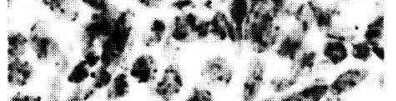

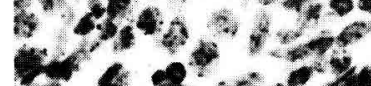
$4+2 x^{2}+42$

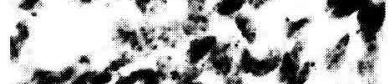

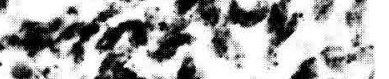

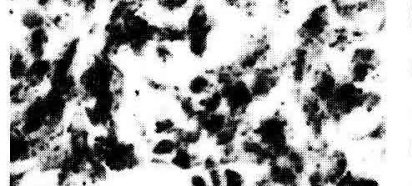

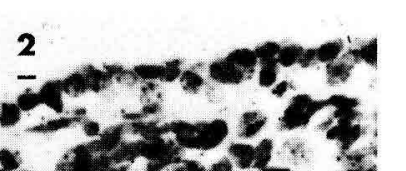

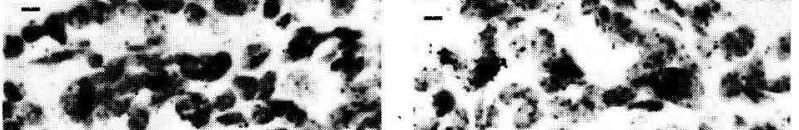

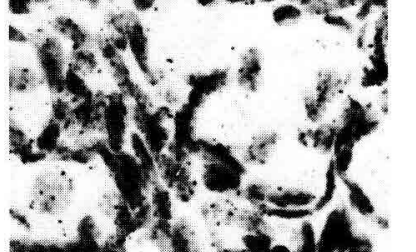
$2 \cdot y^{2}+250$
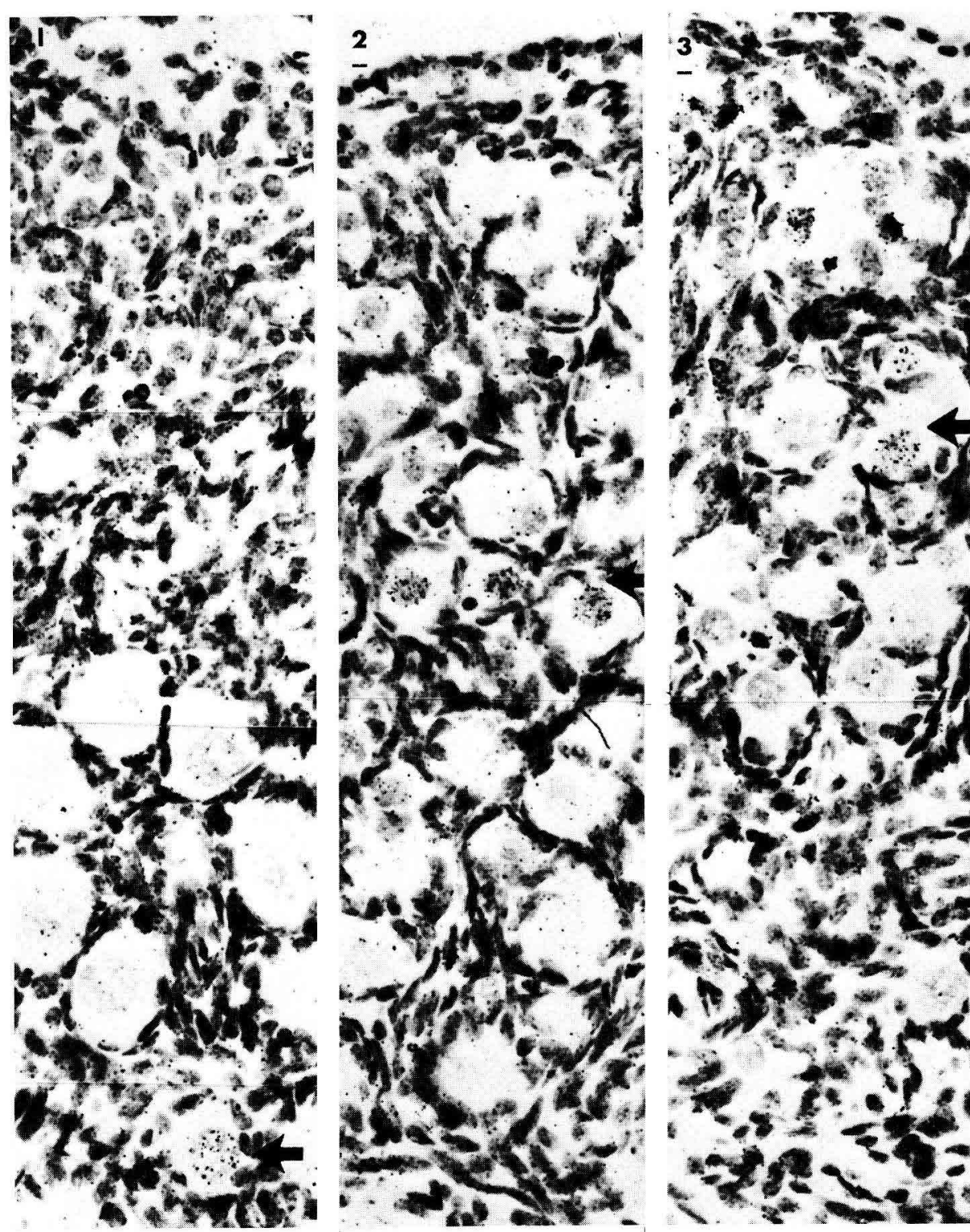

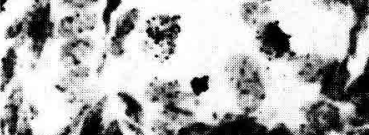

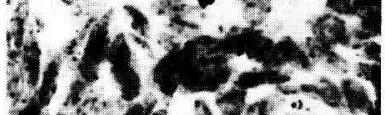
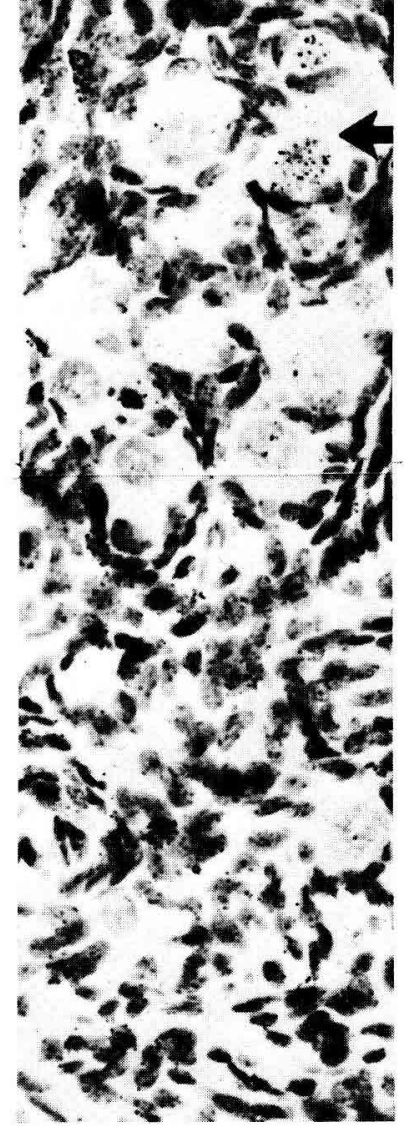


\section{PLANCHE II}

Cultures in vitro associées d'ovaires d'embryon de brebis explantés à des âges différents (49 et 60 jours).

$1^{\circ}$ Grossissement $\times 70$ montrant les deux gonades "cultivées " pendant 10 jours soudées au niveau des flèches.

$2^{\circ}$ Grossissement $\times 285$ montrant :

- partie de gauche : la gonade prélevée avant la "phase critique "sans figures de prophase méïotique à la fin de la culture in vitro,

- partie de droite : la gonade prélevée après la phase critique avec figures de prophase méïotique (quelquesunes sont identifiées sur la planche).

Fixation Bouin Hollande sublimé. Coloration de Feulgen. 

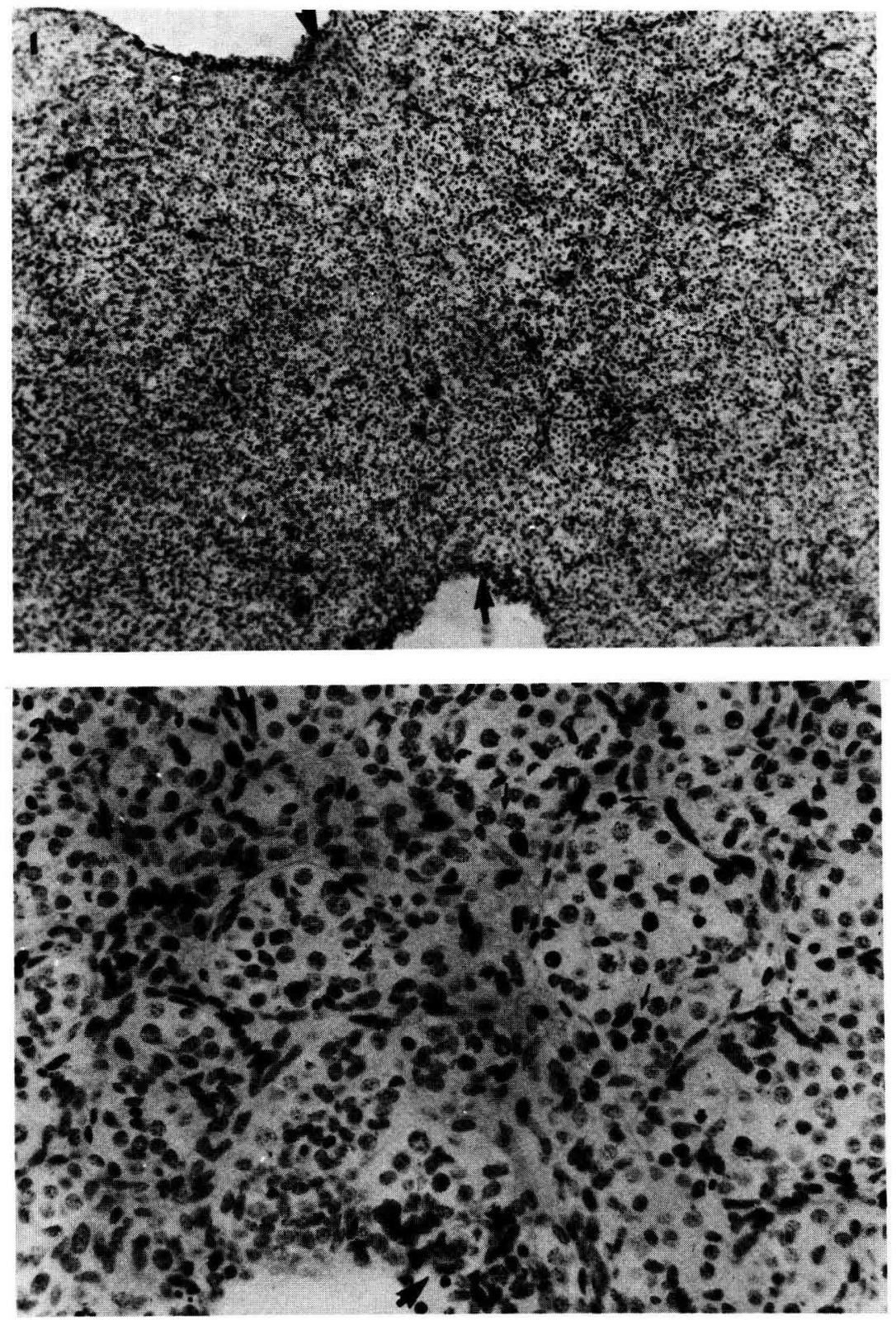

P. MAULÉON 


\section{PLANCHE III}

Aspects cytologiques de noyaux de cellules germinales (Embryon de brebis) (Grossissement $\times 2.700$ ).

Stades ultimes de la méïose atteints en culture in vitro lorsque la gonade femelle est prélevée avant le 50 e jour de la vie de l'embryon. Stades comparables trouvés in vivo.

10 Leptotène en "boule". Ovaire prélevé à 49 jours, mis en culture pendant 8 jours.

$2^{\circ}$ Leptotène de forme semblable trouvé uniquement en fin de période ovogénétique. Embryon de 80 jours.

$3^{\circ}$ Leptotène normal. Ovaire prélevé à 49 jours et mis en culture pendant 8 jours.

$4^{\circ}$ Leptotène normal. Ovaire témoin de 57 jours.

Fixation alcool-acide acétique. Coloration orcéine lactique acétique. 


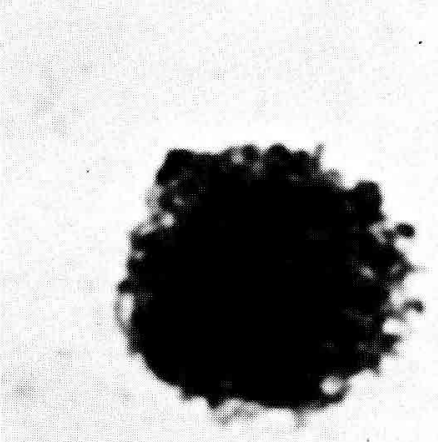 \\ 2}

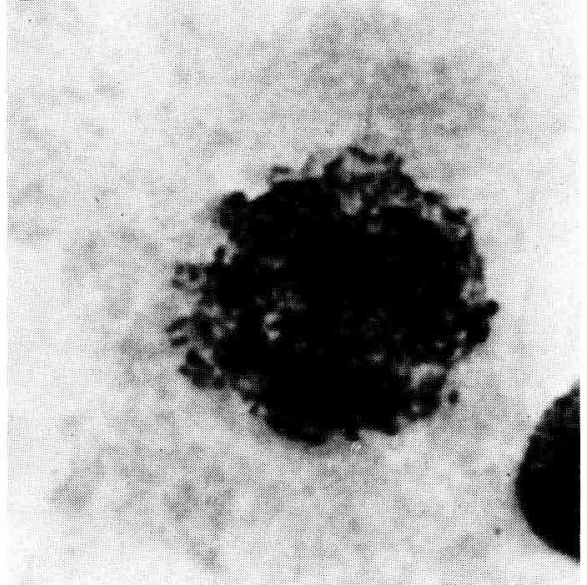

\section{3}

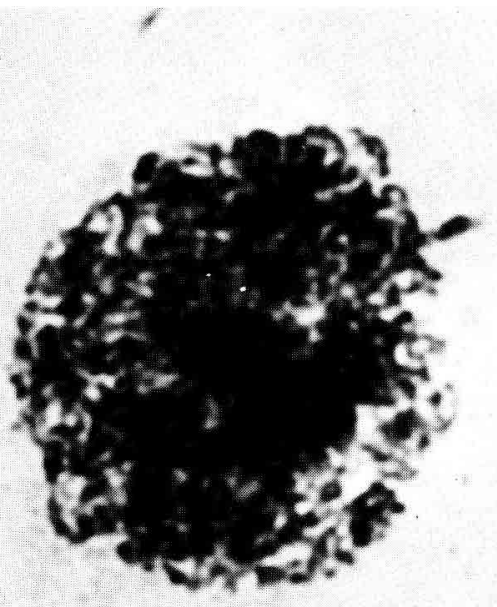

4

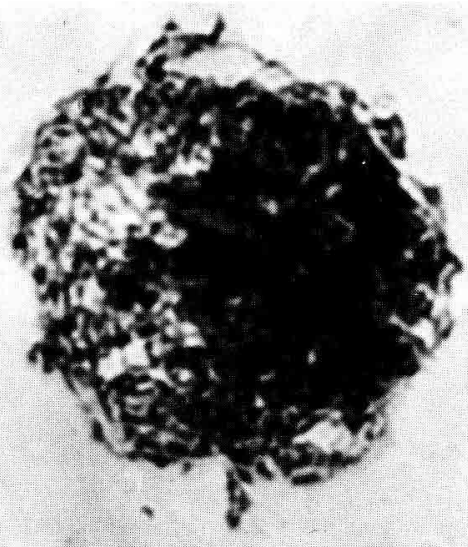

P. MAULÉON 


\section{PLANCHE IV}

Aspects cytologiques de noyaux de cellules germinales (Embryon de brebis) (Grossissement $\times 2.700$ ).

Stades considérés comme antérieurs et postérieurs à ceux trouvés dans les ovaires explantés avant le $50^{\mathrm{e}}$ jour et mis en culture in vitro.

1 et 2 : Stades préleptotène.

3 et 4 : Stades zygotène. Un tel appariement des filaments chromosomiques n'est jamais observé in vitro pour des prélèvements antérieurs à la "période critique".

Fixation alcool-acide acétique. Coloration orcéine lactique acétique. 

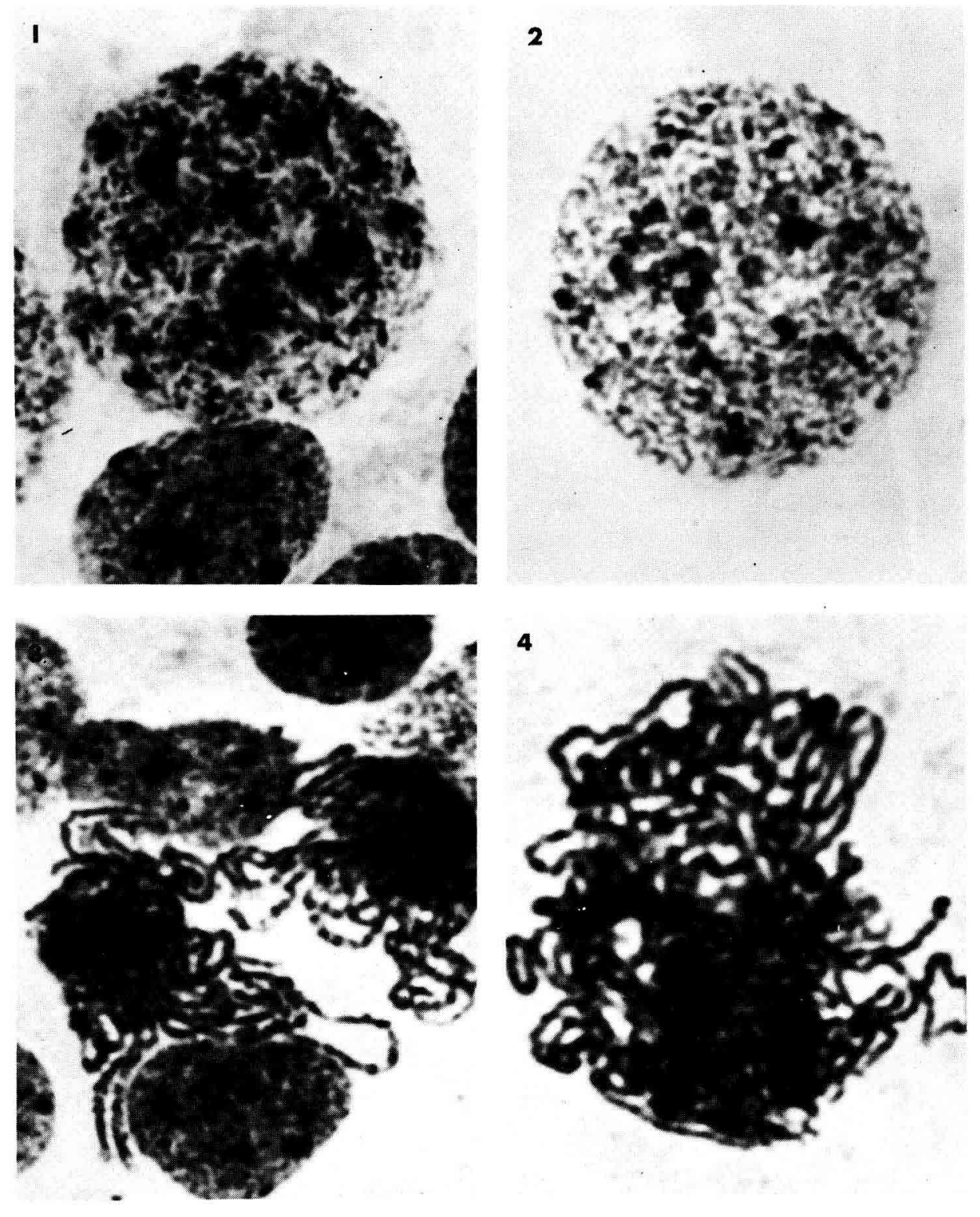